\title{
EFEITO DA TEMPERATURA NA VISCOSIDADE DINÂMICA DOS ÓLEOS LUBRIFICANTES SAE 5W20, SAE 5 W30 E SAE 5 W40
}

César Augusto CANCIAM*

\author{
*Mestre em Engenharia Química, professor lotado no Departamento Acadêmico de Engenharia Química da \\ Universidade Tecnológica Federal do Paraná - Câmpus Ponta Grossa, canciam@utfpr.edu.br
}

Recebido em: 23/10/2013 - Aprovado em: 20/12/2013 - Disponibilizado em: 15/01/2014

RESUMO: Mudanças na viscosidade dinâmica dos óleos lubrificantes afetam diretamente a capacidade de lubrificação e proteção das partes móveis de um motor de combustão interna. O objetivo deste trabalho foi avaliar o efeito da temperatura na viscosidade dinâmica dos óleos lubrificantes SAE 5W20, SAE 5W30 e SAE 5W40 entre 10 e $150^{\circ} \mathrm{C}$, através da estimativa da energia de ativação de fluxo viscoso. Os valores da energia de ativação de fluxo viscoso foram determinados a partir da análise de regressão linear simples dos dados da viscosidade dinâmica em função da temperatura. A energia de ativação de fluxo viscoso dos óleos lubrificantes estudados encontrou-se entre 26,5959 $\mathrm{kJ} \cdot \mathrm{mol}^{-1}$ e $29,5472 \mathrm{~kJ} \mathrm{~mol}^{-1}$. Em todos os casos estudados, os coeficientes de correlação foram próximos da unidade, classificando a correlação linear como muito forte.

PALAVRAS-CHAVE: Lubrificantes. Viscosidade. Energia de ativação de fluxo viscoso. Regressão linear.

\section{EFFECT OF TEMPERATURE ON DYNAMIC VISCOSITY OF LUBRICATING OILS SAE 5W20, SAE 5 W30 E SAE 5 W40}

\begin{abstract}
The changes in the dynamic viscosity of lubricating oils directly affect the ability of lubrication and protection from moving parts of an internal combustion engine. The objective of this work was evaluate the effect of temperature on the dynamic viscosity of lubricating oils SAE 5W20, SAE 5W30 and SAE 5W40 between 10 and $150^{\circ} \mathrm{C}$, by estimating the activation energy of viscous flow. The values of activation energy of viscous flow were determined from the simple linear regression analysis of data of dynamic viscosity in function of temperature. The activation energy of viscous flow of lubricants studied were between $26.5959 \mathrm{~kJ}^{-\mathrm{mol}^{-1}}$ and $29.5472 \mathrm{~kJ} \mathrm{~mol}^{-1}$. In all cases studied, the correlation coefficients were near to unit, classifying as a very strong linear correlation.
\end{abstract}

KEYWORDS: Lubricants. Viscosity. Activation energy of viscous flow. Linear regression.

\section{INTRODUÇÃO}

A viscosidade dinâmica é a propriedade que determina o grau de resistência do fluido a uma força cisalhante. Vários estudos têm demonstrado que essa grandeza é altamente influenciada por mudanças na temperatura, segundo Oliveira, Barros e Rossi (2009).

Brunetti (2008) comenta que a viscosidade dinâmica é diretamente proporcional à força de atração entre as moléculas. Com o aumento da temperatura, essa força de atração diminui, diminuindo também a viscosidade dinâmica.

A redução da viscosidade dinâmica nos líquidos devido ao aumento da temperatura é atribuída ao aumento das distâncias intermoleculares provocadas durante o aquecimento. $\mathrm{O}$ aumento das distâncias intermoleculares reduz as forças de atração entre as moléculas, diminuindo a 
viscosidade dinâmica (GRANJEIRO et al, 2007).

Do ponto de vista da lubrificação, o conhecimento do comportamento da viscosidade dinâmica com a temperatura é importante, pois a viscosidade dinâmica influencia consideravelmente as dimensões da espessura mínima do filme lubrificante que promove a separação das superfícies em contato (PROFITO, 2010).

A Teoria do Estado Ativado de Eyring é a base para a determinação da viscosidade dinâmica nos líquidos. Essa teoria considera que um líquido é constituído por moléculas intercaladas por posições desocupadas, denominadas de vacâncias. Mesmo com o líquido em repouso, as moléculas movem-se com o objetivo de ocuparem as vacâncias adjacentes a elas. A viscosidade dinâmica está relacionada com a força que tende a se opor a esse movimento, sendo uma medida da fricção interna do fluido. Assim, quanto maior a barreira potencial que uma molécula terá que vencer a fim de ocupar a vacância adjacente, maior é a viscosidade dinâmica. Essa barreira potencial é conhecida como energia de ativação de fluxo viscoso, de acordo com Silva, Paredes e Reis (2007).

Bringel, Soares e Soares (2009) comentam que o escoamento de um líquido pode ser considerado como um processo térmico em que as moléculas devem exceder a energia de ativação de fluxo viscoso.
Com relação à energia de ativação de fluxo viscoso, essa grandeza indica a sensibilidade da viscosidade dinâmica devido à mudança de temperatura. Ou seja, quanto maior for a energia de ativação de fluxo viscoso, maior será a influência da temperatura. Altos valores da energia de ativação de fluxo viscoso indicam uma mudança mais rápida na viscosidade dinâmica com a temperatura (SILVA, 2008).

Giap (2010) considera que o efeito da temperatura absoluta $(\mathrm{T})$ sobre a viscosidade dinâmica $(\mu)$ é modelado pela Equação do tipo Arrhenius, conforme a Equação 1.

$$
\mu=\mu_{\infty} \cdot \exp \left(\frac{E_{a}}{R \cdot T}\right)
$$

Em que $E_{a}$ corresponde à energia de ativação de fluxo viscoso; $\mathrm{R}$, à constante universal dos gases ideais e $\mu_{\infty}$, à viscosidade

dinâmica quando a temperatura tende ao infinito.

Com base na Equação 1, Flauzino (2007) comenta que a ordem de grandeza da energia de ativação de fluxo viscoso indica a dependência da viscosidade dinâmica com a temperatura. Dessa forma, os líquidos com altos valores da energia de ativação de fluxo viscoso apresentam grande variação na viscosidade dinâmica em função da temperatura e vice-versa. 
Gratão, Berto e Silveira Júnior (2004) consideram que a Equação 1 pode ser reescrita na forma de:

$$
\ln \mu=\ln \mu_{\infty}+\left(\frac{E_{a}}{R}\right) \cdot \frac{1}{T}
$$

Segundo Canciam (2008), um gráfico de $\ln \mu$ versus $\frac{1}{T}$ fornece uma reta, em que o coeficiente angular corresponde numericamente à razão $\left(\frac{E_{a}}{R}\right)$ e o coeficiente linear, à $\ln \mu_{\infty}$.

O conhecimento da energia de ativação de fluxo viscoso e da viscosidade dinâmica quando a temperatura tende ao infinito permite verificar os efeitos da temperatura sobre a viscosidade dinâmica, além de modelar matematicamente o comportamento reológico desse fluido, comentam Gratão, Berto e Silveira Júnior (2004).

Outra equação que correlaciona a viscosidade dinâmica com a temperatura é a Equação de Vogel, conforme a Equação 3 (PROFITO, 2010).

$$
\mu=a \cdot \exp \left(\frac{b}{T+c}\right)
$$

Em que $a, b$ e $c$ correspondem aos coeficientes empíricos.

Profito (2010) afirma que Equação 3 apresenta resultados bastante precisos para aplicações em engenharia.

O sistema de classificação SAE (realizado pela Society of Automative
Enginners) é o mais antigo sistema adotado nos óleos lubrificantes automotivos para motor e transmissão. Essa classificação define faixas de viscosidade, não levando em conta os requisitos de desempenho (KIMURA, 2010).

O desenvolvimento de aditivos melhoradores de índice de viscosidade possibilitou a fabricação de óleos múltipla graduação, ou também denominados de multiviscosos ou multigraus, como por exemplo, SAE 5W30, SAE 15W40, entre outros. Esses óleos lubrificantes são amplamente usados porque são bastante fluidos em baixas temperaturas (permitindo uma partida mais fácil do motor) e suficientemente espessos a altas temperaturas (possibilitando um desempenho satisfatório), comenta Kimura (2010).

O objetivo deste trabalho foi avaliar o efeito da temperatura na viscosidade dinâmica dos óleos lubrificantes SAE 5W20, SAE $5 \mathrm{~W} 30$ e SAE $5 \mathrm{~W} 40$ entre 10 e $150^{\circ} \mathrm{C}$, através da estimativa da energia de ativação de fluxo viscoso.

\section{MATERIAIS E MÉTODOS}

Para a obtenção da energia de ativação de fluxo viscoso dos óleos lubrificantes SAE 5W20, SAE 5W30 e SAE 5W40 foi considerada a metodologia proposta por Canciam (2012b). 
A Tabela 1 relaciona os valores dos coeficientes empíricos da Equação de Vogel (Equação 3) para os óleos lubrificantes estudados.

Tabela 1 - Coeficientes empíricos na Equação de Vogel para os óleos lubrificantes estudados

\begin{tabular}{cccc}
\hline Coeficientes & SAE 5W20 & SAE 5W30 & SAE 5W40 \\
\hline$a$ (Pa.s) & $3,8896.10^{-5}$ & $5,3550.10^{-5}$ & $1,1220.10^{-4}$ \\
$b\left({ }^{\circ} \mathrm{C}\right)$ & 1224 & 1213,77 & 1018,74 \\
$c\left({ }^{\circ} \mathrm{C}\right)$ & 134,10 & 132,58 & 125,91 \\
\hline
\end{tabular}

Fonte: PROFITO (2010).

A partir da Equação 3 e nos dados indicados na Tabela 1, foram estimadas as viscosidades dinâmicas de cada um dos óleos lubrificantes. Para tanto, foram consideradas as temperaturas de 10, 20, 30, 40, 50, 60, 70, $80,90,100,110,120,130,140$ e $150^{\circ} \mathrm{C}$.

Os valores encontrados para a viscosidade dinâmica do óleo lubrificante SAE 5W20 estão indicados na Tabela 2.

Tabela 2 - Valores estimados da viscosidade dinâmica para o óleo lubrificante SAE 5W20

\begin{tabular}{cc}
\hline Temperatura $\left({ }^{\circ} \mathrm{C}\right)$ & Viscosidade dinâmica $\left(.10^{-2} \mathrm{~Pa} . \mathrm{s}\right)$ \\
\hline 10 & 19,0041 \\
20 & 10,9512 \\
30 & 6,7492 \\
40 & 4,3973 \\
50 & 3,0015 \\
60 & 2,1310 \\
70 & 1,5646 \\
80 & 1,1824 \\
90 & 0,9161 \\
100 & 0,7255 \\
110 & 0,5856 \\
120 & 0,4807 \\
130 & 0,4006 \\
140 & 0,3383 \\
150 & 0,2891 \\
\hline
\end{tabular}

Fonte: Autor.
A Tabela 3 indica os valores da viscosidade dinâmica do óleo lubrificante SAE 5W30. Enquanto que a Tabela 4 indica os valores da viscosidade dinâmica do óleo lubrificante SAE 5W40.

Tabela 3 - Valores estimados da viscosidade dinâmica para o óleo lubrificante SAE 5W30

\begin{tabular}{cc}
\hline Temperatura $\left({ }^{\circ} \mathrm{C}\right)$ & Viscosidade dinâmica $\left(.10^{-2} \mathrm{~Pa} . \mathrm{s}\right)$ \\
\hline 10 & 26,6604 \\
20 & 15,2602 \\
30 & 9,3554 \\
40 & 6,0700 \\
50 & 4,1295 \\
60 & 2,9240 \\
70 & 2,1422 \\
80 & 1,6161 \\
90 & 1,2504 \\
100 & 0,9891 \\
110 & 0,7976 \\
120 & 0,6543 \\
130 & 0,5449 \\
140 & 0,4599 \\
150 & 0,3928 \\
\hline
\end{tabular}

Fonte: Autor.

Tabela 4 - Valores estimados da viscosidade dinâmica para o óleo lubrificante SAE 5W40

\begin{tabular}{cc}
\hline Temperatura $\left({ }^{\circ} \mathrm{C}\right)$ & Viscosidade dinâmica $\left(.10^{-2}\right.$ Pa.s $)$ \\
\hline 10 & 20,1991 \\
20 & 12,0844 \\
30 & 7,7222 \\
40 & 5,2083 \\
50 & 3,6737 \\
60 & 2,6904 \\
70 & 2,0340 \\
80 & 1,5800 \\
90 & 1,2565 \\
100 & 1,0196 \\
110 & 0,8422 \\
120 & 0,7066 \\
130 & 0,6010 \\
140 & 0,5174 \\
150 & 0,4503 \\
\hline
\end{tabular}

Fonte: Autor.

Para a obtenção da razão $\frac{E_{a}}{R}$ e do parâmetro $\ln \mu_{\infty}$ foi realizada a análise de 
regressão linear simples dos dados de $\ln \mu$ versus $\frac{1}{T}$. Para essa análise foram considerados os valores da viscosidade dinâmica indicados nas Tabelas 2, 3 e 4.

Com relação à temperatura, para o cálculo de $\frac{1}{T}$, as temperaturas de 10 a $150^{\circ} \mathrm{C}$ foram convertidas para a escala de temperatura Kelvin. A relação entre as escalas de temperatura encontra-se indicada pela Equação 4.

$$
T(K)=T\left({ }^{\circ} C\right)+273,15
$$

A Equação 5 determinou a razão $\frac{E_{a}}{R}$.

$$
\frac{E_{a}}{R}=\frac{(n \cdot C)-D}{(n \cdot F)-G}
$$

Em que:

$$
\begin{aligned}
C & =\sum\left(\frac{1}{T} \cdot \ln \mu\right) \\
D & =\left[\sum\left(\frac{1}{T}\right)\right] \cdot\left(\sum \ln \mu\right) \\
F & =\sum\left(\frac{1}{T}\right)^{2} \\
G & =\left[\sum\left(\frac{1}{T}\right)\right]^{2}
\end{aligned}
$$

O parâmetro $\ln \mu_{\infty}$ foi obtido através da Equação 10.

$$
\ln \mu_{\infty}=\frac{(n \cdot F \cdot H)-(D \cdot I)}{(n \cdot F)-G}
$$

Em que:

$$
H=\sum \ln \mu
$$

$$
I=\sum\left(\frac{1}{T}\right)
$$

As Equações 5, 6, 7, 8, 9, 10, 11 e 12 são adaptações do trabalho de Canciam (2012b).

O coeficiente de correlação $\left(r^{2}\right)$ para a análise de regressão linear simples dos dados de $\ln \mu$ versus $\frac{1}{T}$ foi obtido através da Equação 13, que também é uma adaptação do trabalho de Canciam (2012b).

$$
r^{2}=\frac{(n \cdot C)-D}{\left\{\sqrt{\left.[(n \cdot F)-G] \cdot \sqrt{\left[(n \cdot J)-H^{2}\right.}\right]}\right\}}
$$

Em que:

$$
J=\sum(\ln \mu)^{2}
$$

De acordo com as Tabelas 2, 3 e 4, $n$ equivale ao número de dados amostrais emparelhados, ou seja, $n$ correspondeu a 15.

A energia de ativação de fluxo viscoso $\left(E_{a}\right)$ foi determinada pela multiplicação da razão $\frac{E_{a}}{R}$ por $R$. Para esse cálculo, foi considerado que a constante universal dos gases ideais é igual a $8,314 \cdot 10^{-3} \mathrm{~kJ} \cdot \mathrm{mol}^{-1} \cdot \mathrm{K}^{-1}$. 


\section{RESULTADOS E DISCUSSÃO}

A Figura 1 ilustra o gráfico de $\mu$

versus $\mathrm{T}$, com base nos dados indicados nas Tabelas 2, 3 e 4 .

Com base na Figura 1, pode-se observar que a viscosidade dinâmica de cada um dos óleos lubrificantes estudados tende a sofrer um decréscimo com a elevação da temperatura, variando inversamente com essa grandeza, ou seja, a elevação da temperatura ocasiona a redução da viscosidade dinâmica.

Tal comportamento também foi observado por Costa (2006) que realizou um estudo da viscosidade dinâmica do óleo bruto de duas cultivares de mamona em função da temperatura.

Figura 1: Viscosidade dinâmica dos óleos lubrificantes estudados em função da temperatura

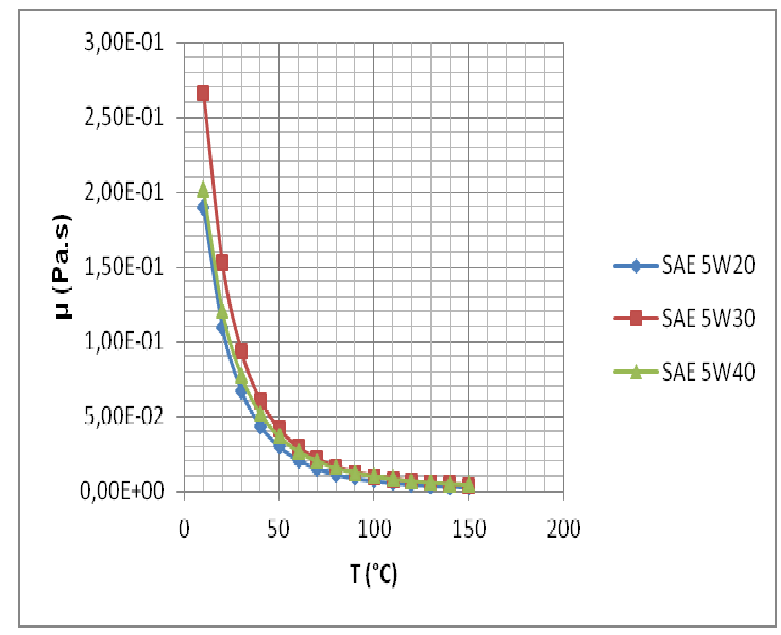

Fonte: Autor.
A Tabela 5 indica os resultados obtidos através da análise de regressão linear simples dos dados de $\ln \mu$ versus $\frac{1}{T}$.

A Tabela 6 indica os valores da energia de ativação de fluxo viscoso $\left(E_{a}\right) \mathrm{e}$ do parâmetro $\mu_{\infty}$ para os óleos lubrificantes estudados.

Tabela 5 - Resultados da análise de regressão linear simples

\begin{tabular}{cccc}
\hline Lubrificante & $\ln \mu_{\infty}$ & $\frac{E_{a}}{R}$ & $r^{2}$ \\
& \multicolumn{3}{r}{} \\
\hline SAE 5W20 & $-14,3315$ & 3528,5056 & 0,9971 \\
SAE 5W30 & $-14,0885$ & 3553,9140 & 0,9970 \\
SAE 5W40 & $-13,1086$ & 3198,9336 & 0,9964 \\
\hline Fonte: Autor. & &
\end{tabular}

Tabela 6 - Valores da energia de ativação de fluxo viscoso e do parâmetro $\mu_{\infty}$

\begin{tabular}{ccc}
\hline Lubrificante & $\mu_{\infty}(\mathrm{Pa} . \mathrm{s})$ & $E_{a}\left(\mathrm{~kJ} . \mathrm{mol}^{-1}\right)$ \\
\hline SAE 5W20 & $5,9691.10^{-7}$ & 29,3360 \\
SAE 5W30 & $7,6110.10^{-7}$ & 29,5472 \\
SAE 5W40 & $2,0277.10^{-6}$ & 26,5959 \\
\hline
\end{tabular}

Fonte: Autor.

Pode-se observar na Tabela 6 que o óleo lubrificante SAE5W30 apresentou a maior energia de ativação de fluxo viscoso em relação aos demais óleos lubrificantes estudados. Dessa forma, os resultados sugerem que a viscosidade dinâmica desse óleo é relativamente mais sensível à mudança de temperatura, quando comparado aos demais óleos lubrificantes estudados. 
De acordo com Silva, Paredes e Reis (2007), a Teoria do Estado Ativado de Eyring considera que quanto maior a energia de ativação de fluxo viscoso, maior é a viscosidade dinâmica.

Neste sentido, comparando os valores da viscosidade dinâmica indicados nas Tabelas 2, 3 e 4, pode-se observar que nas temperaturas $10,20,30,40,50,60,70$ e $80^{\circ} \mathrm{C}$, o valor da viscosidade dinâmica do óleo lubrificante SAE 5W30 é maior em relação aos demais óleos lubrificantes.

A Figura 2 ilustra o gráfico de $\ln \mu$ versus $\frac{1}{T}$.

Figura 2: Gráfico de $\ln \mu$ versus $\frac{1}{T}$ para os óleos lubrificantes estudados

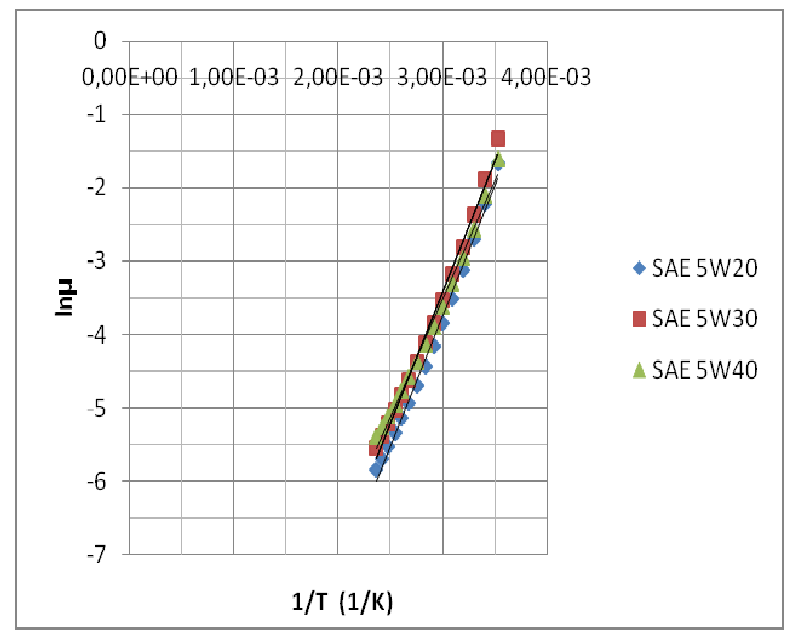

Fonte: Autor.

Com relação ao coeficiente de correlação $\left(r^{2}\right)$, Pinheiro e coautores (2009) comentam que esse parâmetro mede a interdependência linear entre as variáveis e avalia a qualidade do ajuste. De maneira que, quanto mais próximo o coeficiente de correlação for da unidade, melhor é o ajuste da reta em relação aos pontos da dispersão.

Lira (2004) fornece uma classificação para as correlações lineares. A correlação linear é classificada como muito forte, quando os valores do coeficiente de correlação são maiores ou iguais a 0,90 e menor que 1,0 .

Dessa forma, observa-se na Tabela 5 que para todos os óleos lubrificantes estudados, a correlação linear é classificada como muito forte.

Skoog e coautores (2006) comentam que a partir de um modelo de regressão linear, pode-se utilizar a análise de variância (ANOVA) para verificar quanto a reta de regressão "explica" os valores observados que foram utilizados para o ajuste.

A Tabela 7 fornece a análise de variância (ANOVA) para o óleo lubrificante SAE 5W20. Enquanto que as Tabelas 8 e 9 fornecem, respectivamente, as análises de variância para os óleos lubrificantes SAE 5W30 e SAE 5W40. Em cada uma dessas tabelas, GL corresponde aos graus de liberdade; SQM, à soma dos quadrados médios; QM, ao quadrado médio e Fcal, ao valor de F calculado.

Tabela 7 - ANOVA para o óleo lubrificante SAE $5 \mathrm{~W} 20$

\begin{tabular}{ccccc}
\hline $\begin{array}{c}\text { Fonte de } \\
\text { variação }\end{array}$ & GL & SQM & QM & Fcalc \\
\hline Regressão & 1 & 23,9597 & 23,9597 & 2206,9 \\
Resíduos & 13 & 0,1411 & 0,0109 &
\end{tabular}


Fonte: Autor.

Tabela 8 - ANOVA para o óleo lubrificante SAE $5 \mathrm{~W} 30$

\begin{tabular}{ccccc}
\hline $\begin{array}{c}\text { Fonte de } \\
\text { variação }\end{array}$ & GL & SQM & QM & Fcalc \\
\hline Regressão & 1 & 24,3060 & 24,3060 & 2125,8 \\
Resíduos & 13 & 0,1486 & 0,0114 & \\
Total & 14 & 24,4546 & & \\
\hline
\end{tabular}

Fonte: Autor.

Tabela 9 - ANOVA para o óleo lubrificante SAE $5 \mathrm{~W} 40$

\begin{tabular}{ccccc}
\hline $\begin{array}{c}\text { Fonte de } \\
\text { variação }\end{array}$ & GL & SQM & QM & Fcalc \\
\hline Regressão & 1 & 19,693 & 19,6929 & 1803,3 \\
Resíduos & 13 & 0,142 & 0,0109 & \\
Total & 14 & 19,835 & & \\
\hline
\end{tabular}

Fonte: Autor.

De acordo com Triola (2008), no modelo de regressão linear simples a função do teste de $\mathrm{F}$ é a de testar a significância do efeito de $\mathrm{X}$ sobre $\mathrm{Y}$, ou seja, testar a significância do efeito da temperatura sobre a viscosidade dinâmica.

Considerando um nível de significância de 5\%, o valor de Ftab (F tabelado) equivale a 4,67 (TRIOLA, 2008).

Como nas Tabelas 7, 8 e 9 os valores de Fcal são maiores que o valor de Ftab, os resultados da ANOVA sugerem que a regressão linear como um todo faz sentido, ou seja, a variável explicativa temperatura influencia globalmente a variável explicada viscosidade dinâmica.

A Tabela 10 relaciona os valores de $\mathrm{p}$ para os óleos lubrificantes estudados.
Tabela 10 - Valores de p para os óleos lubrificantes estudados

Fonte: Autor.

\begin{tabular}{cc}
\hline Lubrificante & $\mathrm{p}$ \\
\hline SAE 5W20 & $6,718.10^{-16}$ \\
SAE 5W30 & $8,557.10^{-16}$ \\
SAE 5W40 & $2,477.10^{-15}$ \\
\hline
\end{tabular}

Como toda probabilidade, o valor de $\mathrm{p}$ irá variar entre 0 e 1 . Na maioria das áreas, admite-se um valor crítico de $\mathrm{p}$ menor ou igual a 0,05. Dessa forma, assume-se como margem de segurança $5 \%$ de chance de erro, ou $95 \%$ de chance de estar certo, comentam Ara, Musetti e Schneiderman (2003).

Na Tabela 10, pode-se observar que os valores de $\mathrm{p}$ são pequenos, sugerindo que a margem de segurança de chance de erro é pequena.

Foi realizada uma pesquisa na literatura e constatou-se a ausência de valores experimentais para a energia de ativação de fluxo viscoso dos óleos lubrificantes SAE 5W20, SAE 5W30 e SAE 5W40.

A Tabela 11 relaciona a energia de ativação de fluxo viscoso de alguns óleos lubrificantes encontrados na literatura.

Tabela 11 - Valores da energia de ativação de fluxo viscoso de alguns óleos lubrificantes

\begin{tabular}{ccc}
\hline Lubrificante & $E_{a}\left(\mathrm{~kJ}^{\mathrm{mol}}{ }^{-1}\right)$ & Referência \\
\hline SAE 20W30 & 31,1385 & Canciam (2012a) \\
SAE 20W40 & 34,6661 & Canciam (2012a) \\
SAE 30 & 34,7391 & Canciam (2012b) \\
SAE 20W50 & 35,8579 & Canciam (2012a) \\
SAE 40 & 38,1429 & Canciam (2012b) \\
SAE 50 & 39,6802 & Canciam (2012b) \\
\hline Fonte: Autor. & &
\end{tabular}


Comparando a Tabela 6 com a Tabela 11, pode-se observar que os valores da energia de ativação de fluxo viscoso dos óleos lubrificantes SAE 5W20, SAE 5W30 e SAE 20W40 são menores em relação ao óleo lubrificante SAE 20W30.

As diferenças nos valores da energia de ativação de fluxo viscoso podem estar associadas às diferentes composições dos óleos lubrificantes, pois a viscosidade dinâmica é proporcional à força de atração entre as moléculas.

De acordo com Guimarães (2006), os óleos lubrificantes utilizados em motores de combustão interna são constituídos pelos óleos básicos e aditivos.

Os óleos básicos são hidrocarbonetos derivados de petróleo (frações predominantemente de 20 a 25 átomos de carbono) de características parafínicas $(70 \%$ de parafinas ramificadas), naftênicas (20\% de cicloparafinas) e aromáticas

(GUIMARÃES, 2006).

$\mathrm{Na}$ categoria de aditivos encontram-se antioxidantes, desativadores metálicos, antiespumantes, antiferrugens, anticorrosivos, antidesgastes, agentes de extrema pressão, dispersantes/detergentes, melhoradores de índice de viscosidade, abaixadores do ponto de fluidez, bactericidas e corantes (CERQUEIRA, 2004).

Os hidrocarbonetos são substâncias apolares (BRUICE, 2006).
Quando duas moléculas apolares se aproximam uma da outra, suas nuvens de elétrons se repelem, por apresentarem cargas de mesmo sinal. Essa repulsão desloca a nuvem de elétrons de cada molécula. O deslocamento resulta na concentração momentânea da nuvem em uma parte da molécula e, consequentemente, uma diminuição dessa nuvem em outra parte. Assim, a região para onde essa nuvem está mais deslocada apresenta um carga parcial mais negativa. Enquanto que a outra região se torna mais positiva. Isso provoca uma separação temporária de cargas resultando em um dipolo temporário ou induzido (BROWN e HOLME, 2009).

Uma vez formado esse dipolo, seu polo positivo irá atrair os elétrons de outra molécula próxima, que também passará a apresentar um dipolo induzido. Dessa forma, as moléculas irão se atraindo, sucessivamente (BRUICE, 2006).

As forças de atração entre dipolos temporários são chamadas de forças de London e são muito fracas se comparadas às forças entre dipolos permanentes (BROWN e HOLME, 2009).

Segundo Bruice (2006), as forças de London apresentam raio de ação curto, atuando unicamente entre regiões de uma e outra molécula, que se encontram em contato mútuo, ou seja, atuam apenas entre as superfícies das moléculas. Assim, quanto maior o tamanho da molécula, maior a área 
superficial de contato e maior a atuação das forças de London.

\section{CONCLUSÃO}

O objetivo deste trabalho foi avaliar o efeito da temperatura na viscosidade dinâmica dos óleos lubrificantes SAE 5W20, SAE $5 \mathrm{~W} 30$ e SAE $5 \mathrm{E} 40$ entre 10 e $150^{\circ} \mathrm{C}$, através da estimativa da energia de ativação de fluxo viscoso.

Os valores encontrados para a energia de ativação de fluxo viscoso foram de 29,3360 kJ.mol ${ }^{-1}$ (para o lubrificante SAE 5W20), 29,5472 kJ.mol ${ }^{-1}$ (para o lubrificante SAE 5W30) e 26,59598 kJ.mol ${ }^{-1}$ (para o lubrificante SAE 5W40).

Dessa forma, os resultados sugerem que a viscosidade dinâmica do óleo lubrificante SAE 5W30 é relativamente mais sensível à mudança de temperatura, quando comparado aos demais óleos lubrificantes estudados.

Em comparação com outros óleos lubrificantes, os lubrificantes estudados apresentam valores menores de energia de ativação de fluxo viscoso.

Diferenças nos valores da energia de ativação de fluxo viscoso podem estar associadas com as diferenças em termos de composição dos óleos lubrificantes.

Para todos os óleos lubrificantes estudados, a correlação linear foi classificada como muito forte.
A análise de variância revelou que a regressão linear simples, como um todo, faz sentido, pois a temperatura influencia a viscosidade dinâmica dos óleos lubrificantes estudados.

\section{REFERÊNCIAS}

ARA, A.; MUSETTI, A. B.; SCHNEIDERMAN, B. Introdução à Estatística. Edgard Blücher, São Paulo, 2003. $162 \mathrm{p}$.

BRINGEL, R. M.; SOARES, S. A.; SOARES, J. B. Análise de susceptibilidade térmica de ligantes asfálticos através da energia de ativação de fluxo. Transportes, v.17, n.1, p.46-52, 2009.

BROWN, L. S.; HOLME, T. A. Química Geral Aplicada à Engenharia. Cengage Learning, São Paulo, 2009. 653 p.

BRUICE, P. Y. Química Orgânica - volume 1. Pearson Education, São Paulo, 2006. 641p.

BRUNETTI, F. Mecânica dos Fluidos. Pearson Education, São Paulo, 2008. 431 p.

CANCIAM, C. A. Efeito da temperatura na viscosidade de soluções de sacarose. In: SANTOS JÚNIOR, G.; ALMEIDA, D. M.; MICHALOSKI, A. O. (Eds.). Série em Ciência e Tecnologia de Alimentos: agroindústria, energia e meio ambiente. Ponta Grossa: UTFPR, 2008.

CANCIAM, C. A. Estimativa da energia de ativação de fluxo dos óleos lubrificantes SAE 20W30, SAE 20W40 e SAE 20W50. CIATEC - UPF, v.4, n.2, p.42-50, 2012a.

CANCIAM, C. A. Estimativa da energia de ativação de fluxo dos óleos lubrificantes SAE 30, SAE 40 e SAE 50. Revista da 
Universidade Vale do Rio Verde, v.10, n.2, p.202-210, 2012b.

CERQUEIRA, C. P. Estudo do reaproveitamento energético de óleos lubrificantes usados. Dissertação de Mestrado em Regulação da Indústria de Energia da Universidade Salvador - UNIFACS, 2004. $104 \mathrm{p}$.

COSTA, T. L. Características físicas e físicoquímicas do óleo de duas cultivares de mamona. Dissertação de Mestrado em Armazenamento e Processamento de Produtos Agrícolas da Universidade Federal de Campina Grande, 2006. 113 p.

FLAUZINO, R. D. Influência da temperatura e do teor de gordura nos parâmetros reológicos do leite condensado e creme de leite. Dissertação de Mestrado em Engenharia e Ciência de Alimentos da Universidade Estadual Paulista "Júlio de Mesquita Filho", 2007. 100 p.

GIAP, S. G. E. The hidden property of Arrhenius-type relationship: viscosity as a function of temperature. Journal of Physical Science, v.21, n.1, p.29-39, 2010.

GRANJEIRO, A. A.; QUEIROZ, A. J. M.; FIGUEIRÊDO, R. M. F.; MATA, M. E. R. M. C. Viscosidades de polpas concentradas de figo-da-Índia. Revista Brasileira de Agrociência, v.13, n.2, p.219-224, 2007.

GRATÃO, A. C. A.; BERTO, M. I.; SILVEIRA JÚNIOR, V. Reologia do açúcar líquido invertido: influência da temperatura na viscosidade. Ciência e Tecnologia de Alimentos, v.24, n.4, p.652-656, 2004.

GUIMARÃES, J. Rerrefino de óleos lubrificantes de motores de combustão interna pelo processo de ultrafiltração e adsorção. Dissertação de Mestrado em Engenharia Ambiental da Universidade do Estado do Rio de Janeiro, 2006. 83 p.
KIMURA, R. K. Uso da técnica de análise de óleo lubrificante em motores diesel estacionários, utilizando-se misturas de biodiesel e diferentes níveis de contaminação do lubrificante. Dissertação de Mestrado em Engenharia Mecânica da Universidade Estadual Paulista "Júlio de Mesquita Filho", 2010. 129 p.

LIRA, S. A. Análise de correlação: abordagem teórica e de construção dos coeficientes com aplicação. Dissertação de Mestrado em Ciências da Universidade Federal do Paraná, 2004. 196p.

SKOOG, D. A.; WEST, D. M.; HOLLER, F. J.; CROUCH, S. R. Fundamentos de Química Analítica. Cengage Learning, São Paulo, 2006. 1124 p.

OLIVEIRA, R. C.; BARROS, S. T. D.; ROSSI, R. M. Aplicação da metodologia Bayesiana para o estudo reológico da polpa de uva. Revista Brasileira de Produtos Agroindustriais, v.11, n.1, p.73-80, 2009.

PINHEIRO, J. I. D.; CUNHA, S. B.; CARVAJAL, S. R.; GOMES, G. C. Estatística básica: a arte de trabalhar com dados. Elsevier, Rio de Janeiro, 2009. 295 p.

PROFITO, F. J. Modelagem unidimensional do regime misto de lubrificação aplicado a superfícies texturizadas. Dissertação de Mestrado em Engenharia Mecânica da Universidade de São Paulo, 2010. 193 p.

SILVA, A. A.; PAREDES, M. L. L.; REIS, R. A. Modelagem de viscosidade de misturas não - eletrolíticas. In: Congresso Brasileiro de Engenharia Química em Iniciação Científica, 8, 2007, São Carlos: Anais... São Carlos: Ufscar, 2007.

SILVA, S. A. Estudo do comportamento reológico dos adesivos hot melt PSA e a sua relação com a composição e as propriedades adesivas. Dissertação de Mestrado em Engenharia Química da Universidade Federal do Rio Grande do Sul, 2008. 123 p. 
SKOOG, D. A.; WEST, D. M.; HOLLER, F. J.; CROUCH, S. R. Fundamentos de Química Analítica. Cengage Learning, São Paulo, 2006. 1124 p.

TRIOLA, M. F. Introdução à Estatísitca.

LTC, Rio de Janeiro, 2008, 720 p. 